\title{
Reabilitação com tratamento de pino intrarradicular de fibra de vidro em pré-molar: relato de caso clínico
}

\author{
Rehabilitation with treatment of fiberglass intraradicular post in premolar: clinical case report \\ Rehabilitación con tratamiento de poste intrarradicular de fibra de vidrio en premolar: reporte de
} caso clínico

Recebido: 19/11/2021 | Revisado: 24/11/2021 | Aceito: 25/11/2021 | Publicado: 04/12/2021

\author{
Michael Pinho de Araújo \\ ORCID: https://orcid.org/0000-0001-6633-9451 \\ Centro Universitário Fametro, Brasil \\ E-mail: michaelpinhodearaujo@gmail.com \\ Regivaldo Pontes da Silva \\ ORCID: https://orcid.org/0000-0003-2992-8059 \\ Centro Universitário Fametro, Brasil \\ E-mail: Regispontes123@gmail.com \\ Gabriel Catunda de Souza \\ ORCID: https://orcid.org/0000-0002-0806-9841 \\ Especialista em Prótese, Brasil \\ E-mail: gabriel.catunda@fametro.edu.br \\ Gabriela de Figueiredo Meira \\ ORCID: https://orcid.org/0000-0002-8285-8769 \\ Universidades Federal de Santa Maria, Brasil \\ E-mail: gabrielameira1@ hotmail.com
}

\begin{abstract}
Resumo
Objetivo: O presente trabalho propõe-se a relatar um acaso clínico, onde em uma paciente do sexo feminino, de 25 anos de idade, foi instalado um pino de fibra de vidro e uma coroa de cerâmica metal free. Metodologia: o presente estudo trata de um relato de caso clínico de cunho analítico descritivo (Pereira et al., 2018), Relato de caso clínico: Paciente Z.S. S do gênero feminino, 25 anos de idade, compareceu à Clínica odontológica do CEUNI- FAMETRO com queixa de insatisfação estética dos dentes posteriores superiores. Durante a anamnese a paciente relata que realizou tratamento endodôntico no elemento 15 e restauração com resina composta, em meados do ano de 2017, após o tratamento a paciente relatou que por volta de 6 meses depois, iniciou uma perda significativa de tecido coronário, na região de faces mesio-oclusal (MO), e ao longo do tempo, avançou para faces da região disto-lingual (DL), onde concluiu a perda e restando apenas a parede vestibular do elemento em questão. Considerações finais: $\mathrm{O}$ presente estudo concluiu que a reabilitação proposta com o uso de pino de fibra de vidro e a coroa metal free, obtiveram excelentes resultados, pois foram capazes de devolver não só a função, mas a saúde e estética para a paciente.
\end{abstract}

Palavras-chave: Cerâmica; Pino; Intrarradicular; Coroa.

\begin{abstract}
Objective: The present study aims to report a clinical chance, where a 25 -year-old female patient was fitted with a fiberglass post and a metal free ceramic crown. Methodology: this study is a clinical case report of descriptive analytical nature (Pereira et al., 2018), Clinical case report: Patient Z.S. S female, 25 years old, attended the CEUNIFAMETRO dental clinic complaining of aesthetic dissatisfaction of the upper posterior teeth. During the anamnesis, the patient reports that she underwent endodontic treatment in element 15 and restoration with composite resin, in mid-2017, after the treatment, the patient reported that around 6 months later, she started a significant loss of coronary tissue in the region from the mesio-occlusal (MO) facets, and over time, it advanced to the distolingual (DL) facets, where the loss ended and only the buccal wall of the element in question remained. Final considerations: The present study concluded that the proposed rehabilitation using a fiberglass post and a metal free crown, obtained excellent results, as they were able to restore not only function, but also health and esthetics for the patient.
\end{abstract}

Keywords: Ceramic; Pin; Intraradicular; Crown.

\section{Resumen}

Objetivo: El presente trabajo tiene como objetivo reportar una oportunidad clínica, donde a una paciente de 25 años se le colocó un poste de fibra de vidrio y una corona de cerámica libre de metal. Metodología: este estudio es un reporte de caso clínico de naturaleza analítica descriptiva (Pereira et al., 2018), Reporte de caso clínico: Paciente Z.S. S mujer de 25 años, acudió a la clínica dental CEUNI-FAMETRO por insatisfacción estética de los dientes posteriores superiores. Durante la anamnesis, la paciente relata que fue sometida a tratamiento endodóntico en el elemento 15 y 
restauración con resina compuesta, a mediados de 2017, luego del tratamiento, la paciente informó que alrededor de 6 meses después, inició una pérdida significativa de tejido coronario en la región. desde las facetas mesiooclusales (MO), y con el tiempo avanzó hasta las facetas distolinguales (DL), donde finalizó la pérdida y solo quedó la pared vestibular del elemento en cuestión. Consideraciones finales: El presente estudio concluyó que la rehabilitación propuesta utilizando un poste de fibra de vidrio y una corona libre de metal, obtuvo excelentes resultados, ya que lograron restaurar no solo la función, sino también la salud y la estética del paciente.

Palabras clave: Cerámica; Alfiler; Intrarradicular; Corona.

\section{Introdução}

Em dentes com grande destruição coronária, especialmente naqueles submetidos ao tratamento endodôntico, a possibilidade de falha biomecânica é grande, podendo decorrer da própria intervenção endodôntica (Amarnath et al., 2015).

A seleção do pino deve ser realizada com muita atenção, considerando alguns fatores diretos como: propriedades mecânicas, design, translucidez e a radiopacidade, que são de extrema importância para o resultado satisfatório final do procedimento restaurador. Além disso, outros fatores indiretos como agente de cimentação, material do núcleo de preenchimento da coroa e qualidade do tratamento endodôntico estão relacionados (Furtos, Baldea \& Silaghi-Dumitrescu, 2016).

O uso de pinos de fibra de vidro tem suas vantagens em relação à estética, pois se apresentam na cor branca, conferindo translucidez e naturalidade às próteses de cerâmica pura de dissilicato de lítio, as quais possuem copings igualmente translúcidos. Também apresentam vantagens no que diz respeito à união à estrutura dental e ao módulo de elasticidade. (Eskitasciolglu et al., 2002; Sirimais et al.,1999).

Nesse sentido, o uso dos retentores intrarradiculares são indicados para dentes com coroas totais ou parcialmente destruídas e que necessitam de tratamento com prótese (Pegoraro, 2012) e auxiliar na melhoria do volume coronário parcialmente destruído e proporcionar ao preparo forma de resistência para o deslocamento da futura restauração (Oliveira, 2013).

Os pinos de fibra são frequentemente utilizados porque contribuem para o suporte e retenção de restaurações diretas e indiretas, sendo considerados uma opção prática e econômica para restaurar os dentes tratados endodonticamente, que sofreram grande perda de estrutura (Bitter et al., 2009).

Os pinos de fibra de vidro podem causar um efeito real de biomimetização do tecido dental de maneira que a força oclusal se espalhe ao longo da raiz, possibilitando alívio do estresse mastigatório (Pedreira, 2013), isto posto, sendo o módulo de elasticidade do pino semelhante ao do tecido dentário auxilia na proteção contra prováveis quebras formadas pelas cargas mastigatórias (Fernandes Junior, 2016).

O uso de pinos intrarradiculares é frequentemente utilizado em casos onde a estrutura remanescente coronária do dente é escassa para promover o suporte necessário ao material restaurador (Kaya, 2013). Sendo que estes são esteticamente favoráveis, pois permitem a passagem de luz, apresentando coloração próxima a da estrutura dental em procedimentos restauradores. (Muniz, 2005).

$\mathrm{O}$ aumento do comprimento do pino não aumentaria a resistência à fratura, sendo acompanhado de um desgaste adicional das paredes do canal, o que poderia, inclusive, diminuir a resistência radicular (Pilo et al., 2008; Büttel et al., 2009).

Existem diversos tipos de cimentos que podem ser utilizados no interior do canal radicular associado aos pinos de fibra de vidro, sendo que os cimentos resinosos são muito utilizados. Estes cimentos são denominados convencionais, quando necessitam de condicionamento ácido, enxágue e aplicação de sistema adesivo antes de sua inserção; e, cimentos resinosos autoadesivos quando dispensam essas etapas citadas (Farina et al., 2011; Nova et al., 2013). 
Estudos revelam que a seleção de materiais de propriedades mecânicas similares à dentina, com capacidade de união à estrutura dental, além de ser biocompatível no ambiente oral são capazes de produzir resultados satisfatórios (Jiang Kongo, 2013).

Diversos estudos relataram que o comprimento ideal do pino, independentemente de seu material, deve ter dois terços do comprimento da raiz, ou ao menos o equivalente ao comprimento da coroa clínica (Fernandes et al., 2003; Cheung, 2005; Alomari et al., 2011).

Em suma, o objetivo deste trabalho foi descrever o caso clínico de reabilitação oral com pino de fibra de vidro e coroa de cerâmica, de forma que o primeiro contribui para um quadro esteticamente favorável ao paciente.

\section{Metodologia}

Partindo da permissão da paciente por meio do Termo de Consentimento Livre e Esclarecido (TCLE) em partilhar sua imagem para devido fim, o presente estudo trata de um relato de caso clínico de cunho analítico descritivo (Pereira et al., 2018), realizado na clínica odontológica do CEUNI Fametro, na disciplina de estágio supervisionado em Clínica Integrada II, do curso de graduação do Centro Universitário Fametro.

\section{Resultados e Discussão}

Paciente Z.S. S do gênero feminino, 25 anos de idade, compareceu à Clínica odontológica do CEUNI- FAMETRO com queixa de insatisfação estética dos dentes posteriores superiores. Durante a anamnese a paciente relata que realizou tratamento endodôntico no elemento 15 e restauração com resina composta, em meados do ano de 2017, após o tratamento a paciente relatou que por volta de 6 meses depois, iniciou uma perda significativa de tecido coronário, na região de faces mesiooclusal (MO), e ao longo do tempo, avançou para faces da região disto-lingual (DL), onde concluiu a perda e restando apenas a parede vestibular do elemento em questão. Não há relato de sintomatologia dolorosa. A paciente não apresenta nenhum comprometimento sistêmico e nem relatos de hipersensibilidade. Ao exame clínico inicial, verificou-se que havia uma perda significativa de coroa dentária do elemento 15 , restando somente face vestibular da coroa dentária desse elemento com escurecimento na região cervical e presença de restauração com resina composta na cúspide. (Figura 1).

Figura 1: A) Fotografia intrabucal lateral direita inicial; B) Fotografia intrabucal frontal.

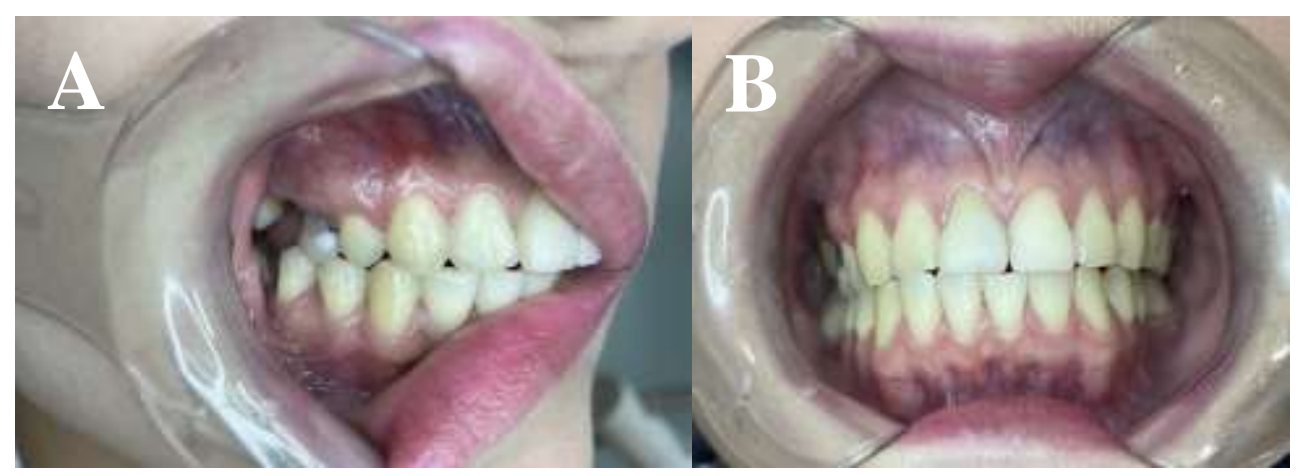

Fonte: Autores.

No exame radiográfico, foi possível observar um tratamento endodôntico satisfatório realizado, também pode ser observado área radio lúcida envolvendo $1 / 3$ da coroa com extensão para mesial, sugestivo de perda de estrutura coronária, também foi verificado reabsorção horizontal da crista alveolar (Figura 3). 
Figura 3: Radiografia periapical do elemento 15.

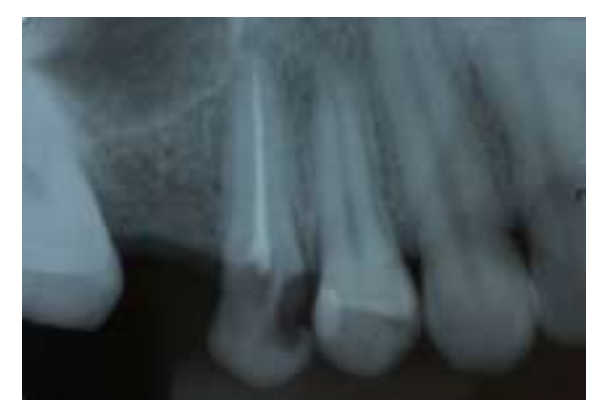

Fonte: Autores.

O planejamento do tratamento do elemento dentário foi primeiramente realizar uma profilaxia intra oral, após foi preciso a administração de anestesia com lidocaína 2\% com epinefrina 1:100.000 nas papilas, uma melhor comodidade para a paciente com relação ao desconforto do grampo, na primeira tentativa de acomodação do grampo, a face vestibular fraturou, nesse momento foi realizado elevação de margem profunda (DME), com resina composta (Filtek® z250 XT, 3M) com objetivo de melhor adaptar o grampo 208 e lençol de borracha (All Prime), para isolamento absoluto (Figura 4) e instalação de pino de fibra de vidro.

Figura 4: Grampo em posição.

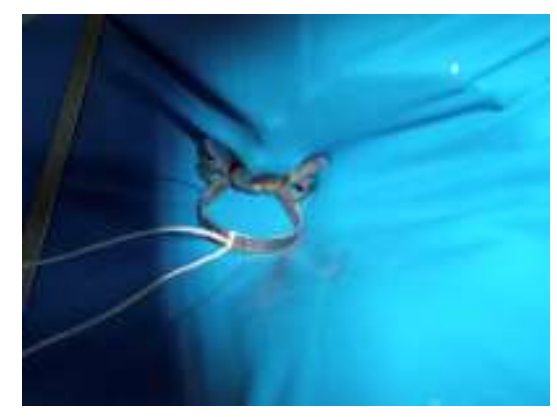

Fonte: Autores.

Por meio da radiografia periapical foi feita a medição do pino de fibra de vidro, onde foi escolhido o pino $\mathrm{n}^{\mathrm{o}} 1.0$ da (Whitepost DC, FGM, Brasil) no tamanho 18 milímetros, sendo o dente 23 milímetros, foi necessário deixar 5 milímetros de obturação do canal até o ápice, dado o início da desobstrução do canal radicular, com brocas Gates $\mathrm{n}^{\circ} 2,3$, (Maillefer Dentsply, USA) e em seguida a utilização da broca alargadora (Whitepost DC, FGM, Brasil), especial para o pino, sempre em rotação baixa e irrigação constante, e o auxílio de um espelho clínico intrabucal (Figura 5).

Figura 5: Alargamento com broca (WHITEPOST DC DA MARCA FGM).

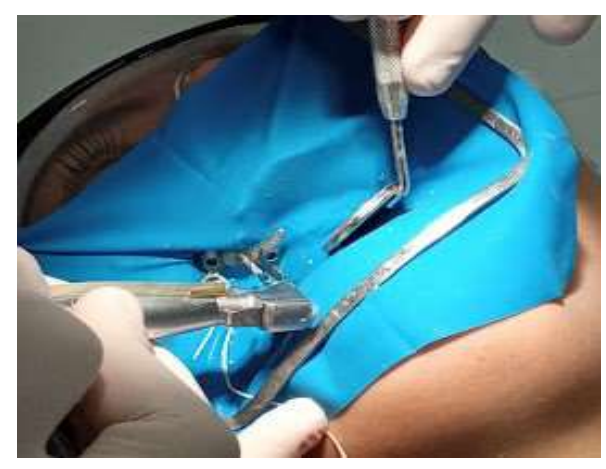

Fonte: Autores. 
Após o preparo do canal, foi realizado a limpeza com soro fisiológico 0,9\%, secado abundantemente com pontas absorventes endodônticas da marca Dentsply, não houve necessidade de ataque ácido do conduto, também não foi realizado a aplicação do adesivo, pois o cimento escolhido para o tratamento, foi o cimento resinoso Dual auto condicionante e autoadesivo A2 (seT PP, SDI, Australia).

O preparo do pino foi realizado, primeiramente submerso em álcool $70 \%$ dentro de um pote dappen, retirado o pino com uma pinça clínica e esperado secar, em seguida realizado a aplicação de silano (Angelus, Brasil) com o auxílio de uma ponta fina de microbrush (Cavibrush, FGM, Brasil), ao término do tempo de volatilização de aproximadamente 1 minuto, uma leve camada de adesivo universal (Ambar, FGM, Brasil) foi aplicada e realizada a fotoativação por 10 segundos, com pino pronto para ser instalado no conduto, logo seguiu-se com a outra fase do tratamento. Com aplicação eficaz do cimento no conduto (figura 6), foi instalado o pino dentro, após retirados os excessos de cimento, esperou-se um tempo variável de 3 a 5 minutos de polimerização, e fotopolimerização por 120 segundos (Figura 7).

Figura 6: A) Aplicação do resinoso Dual A2 (seT PP, SDI, Australia); B) Instalação do pino n ${ }^{\circ} 1.0$ da (Whitepost DC, FGM, Brasil); C) Fotopolimerização.

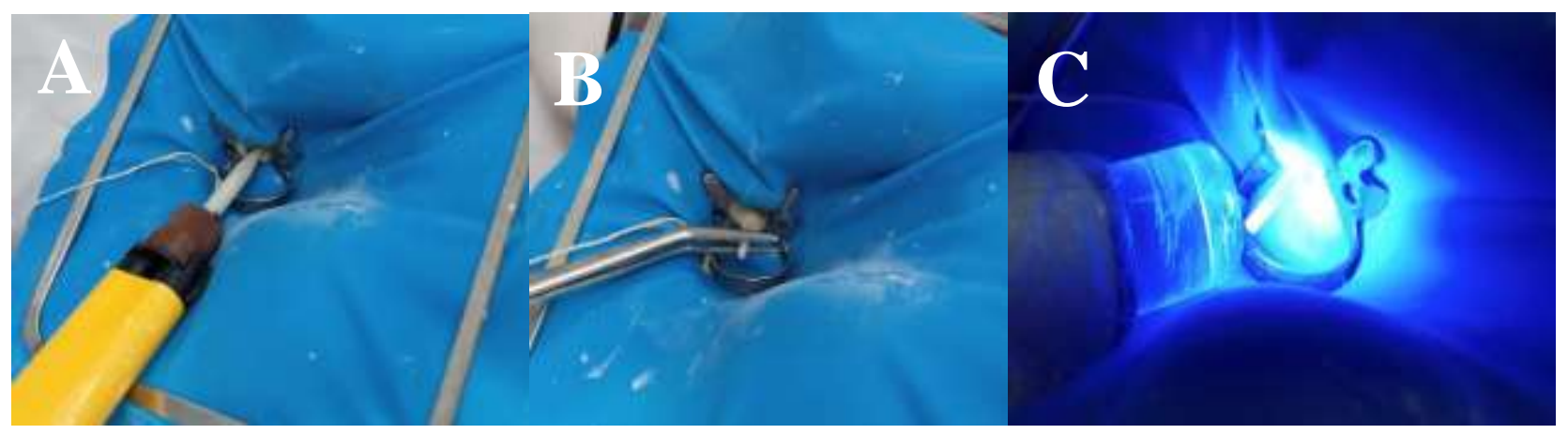

Fonte: Autores.

Após o término da fotopolimerização, foi iniciado o protocolo de preparo para coroa provisória, retirado o isolamento absoluto com tesoura e pinça Palmer, com uma broca 3145 FG(American Burrs, Invicta, Brasil) foi realizado um desgaste chanfrado, e logo dando forma, respeitando a anatomia do elemento e gengiva marginal, também um espaço biológico, para melhor adaptação da coroa, notado uma insuficiência de dente no preparo para moldagem futura, foi marcado cirurgia de aumento de coroa com desgaste ósseo, para melhor moldagem com silicone e assim adaptação da coroa.

Ao término foi feito a outra parte, que seria a confecção da coroa provisória, primeiramente foi isolado o preparo e adjacências, com vaselina sólida e auxílio de um pincel de pelo sintético, com Resina acrílica na Cor 66, (VipiFlash ), foi utilizado a técnica da bolinha e após a manipulação da resina, foi colocado em posição no elemento, foi pedido para a paciente fazer uma mordida, para ficar registrado e assim poder ser feito os desgastes necessários a partir da anatomia copiada das cúspides, realizado os desgastes das arestas da coroa que ficaram em excesso, finalmente a coroa foi cimentada no lugar com cimento provisório (Temp Bond NE, Kerr) (Figura 7 e 8). 
Figura 7: A) Preparo com uma broca 3145 FG (American Burrs, Invicta, Brasil); B) Término do preparo para coroa; C) Isolamento por com vaselina sólida;

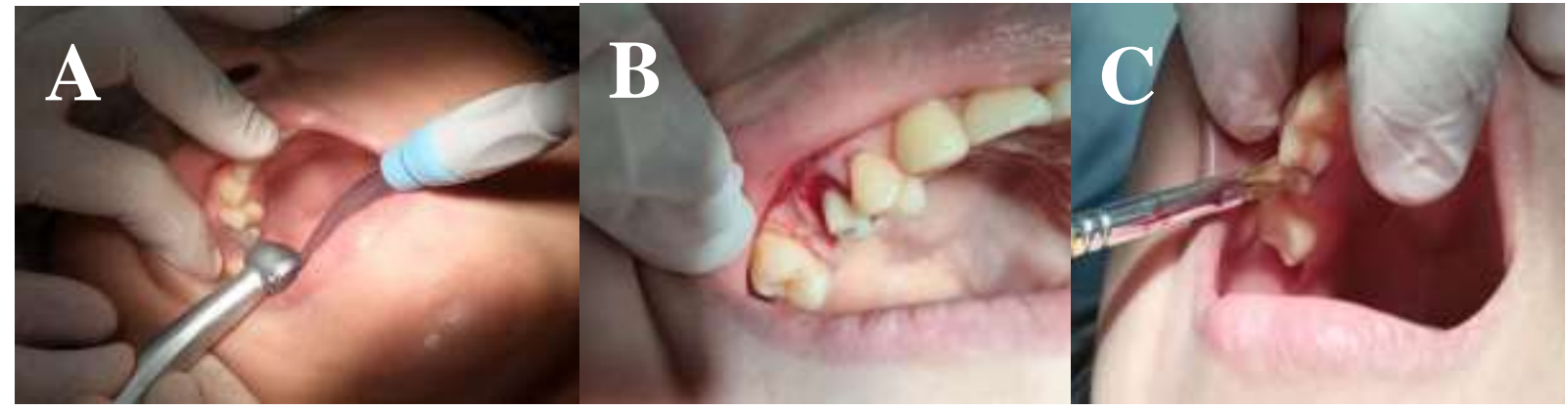

Fonte: Autores.

Figura 8: A) Confecção da coroa provisória pela técnica de bolinha; B) Cimentação da coroa provisória (Temp Bond NE, Kerr).

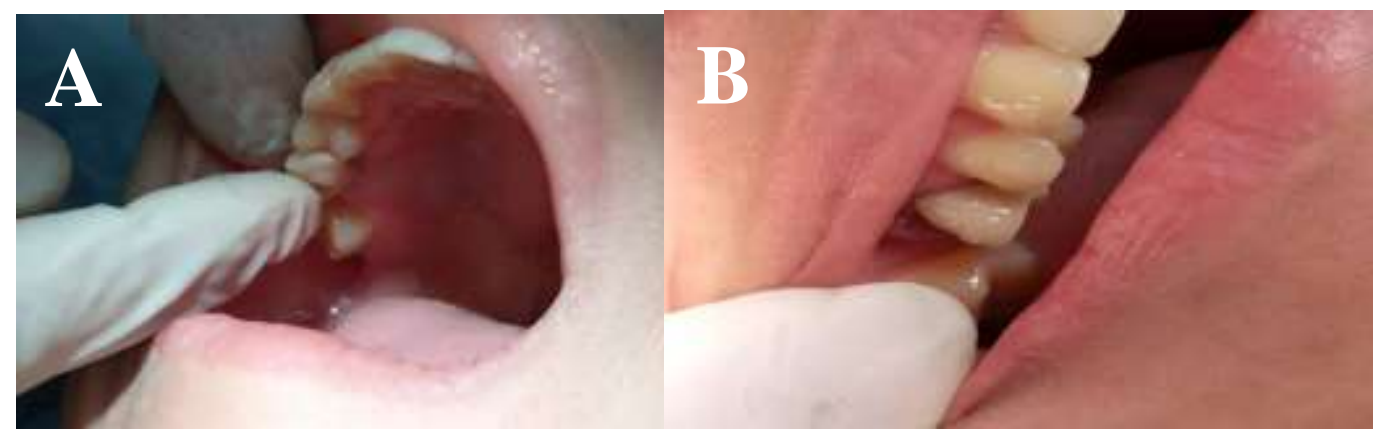

Fonte: Autores.

Realizada cirurgia de aumento de coroa, com desgaste ósseo, com objetivo de melhor adaptação da coroa, realizada com broca esférica cirúrgica $n^{\circ} 4$, após o procedimento a medição com uma sonda milimetrada, mostrou a medida de 3 milímetros, uma sutura simples foi realizada na mesial e distal do elemento, após 15 dias de cicatrização, o tecido da gengiva marginal pode se posicionar adequadamente para ser possível a moldagem (Figura 10)

Figura 10: A) Sondagem pós aumento de coroa clínica; B) Sutura pós aumento de coroa clínica.

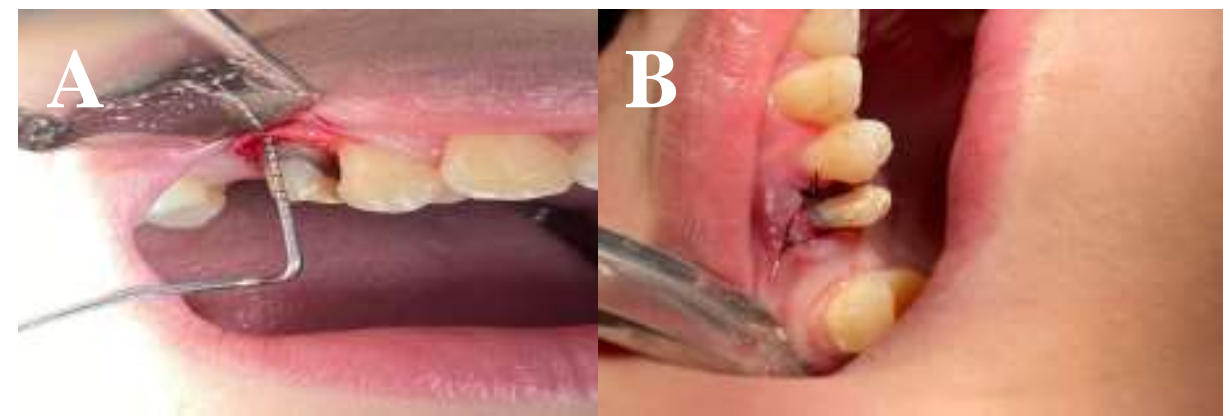

Fonte: Autores.

$\mathrm{Na}$ fase da moldagem para confecção da coroa metal free, foi utilizado uma moldeira metálica de numeração 6 Superior, retirado a coroa provisória, em seguida colocado em posição um fio retrator de numeração 00 ultrapak da marca (ULTRADENT), foi realizado a técnica de moldagem de dois passos, primeiro é manipulado o material pesado do silicone de condensação Perfil (VIGODENT - COLTENE), duas porções na palma da mão, em seguida feito a marcação com o dosador, dentro do círculo se faz um “+” com o catalisador bisnaga, manipulado sempre de fora para dentro, foi constituído uma cor 
homogênea, posto na moldeira e em seguida levado a arcada da paciente, retirado após a presa foi realizado o alívio no molde (Figura 11).

Figura 11: A) Moldagem com silicone (Perfil, Coltene, Brasil); B) Confecção de alívio.

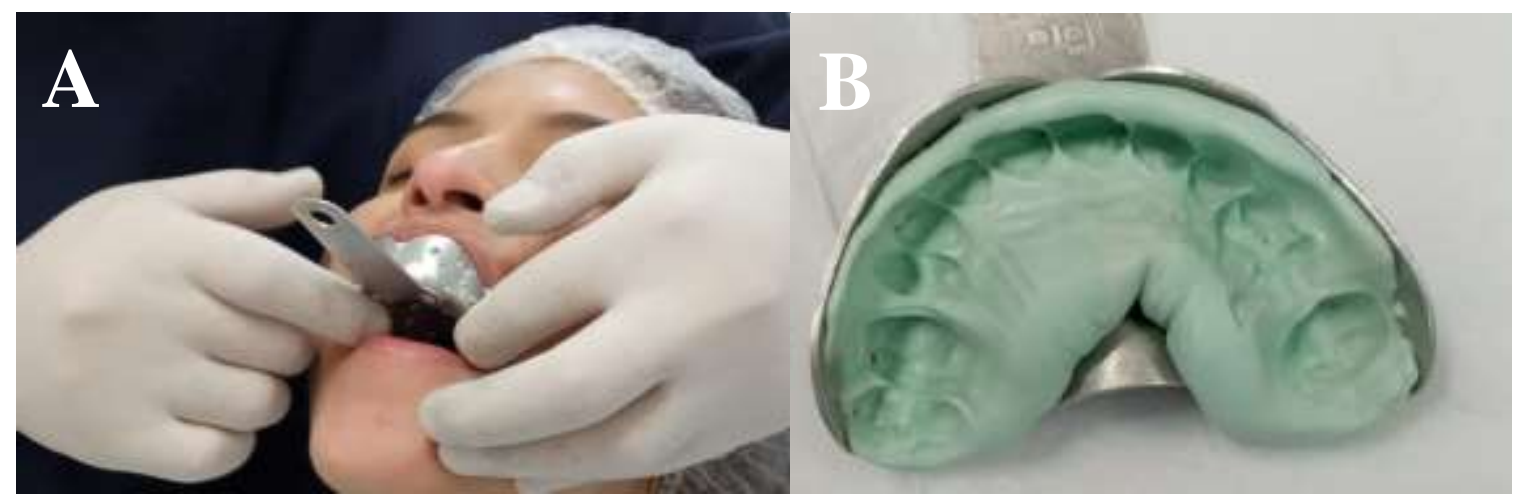

Fonte: Autores.

Após confeccionado o alívio com o auxílio de uma broca Maxicut, o segundo passo foi realizar a proporção catalisador/pasta fluida, em cima de uma placa de vidro , com auxílio de uma espátula $\mathrm{n}^{\circ} 36$ para a manipulação adequada do silicone, nessa fase foi utilizado um injetor de elastômero, assim foi introduzido a porção na seringa uma porção posta na moldagem, e a outra na região de gengiva marginal, no momento em que era retirado o fio retrator gengival, logo foi colocado a moldeira em posição novamente, ao término da presa completa, retirado a moldagem pode se observar uma moldagem mais fiel do preparo (Figura 12).

Figura 12: A) Proporção catalisador/ pasta fluida Perfil (VIGODENT - COLTENE); B) Moldagem $2^{\circ}$ passo.

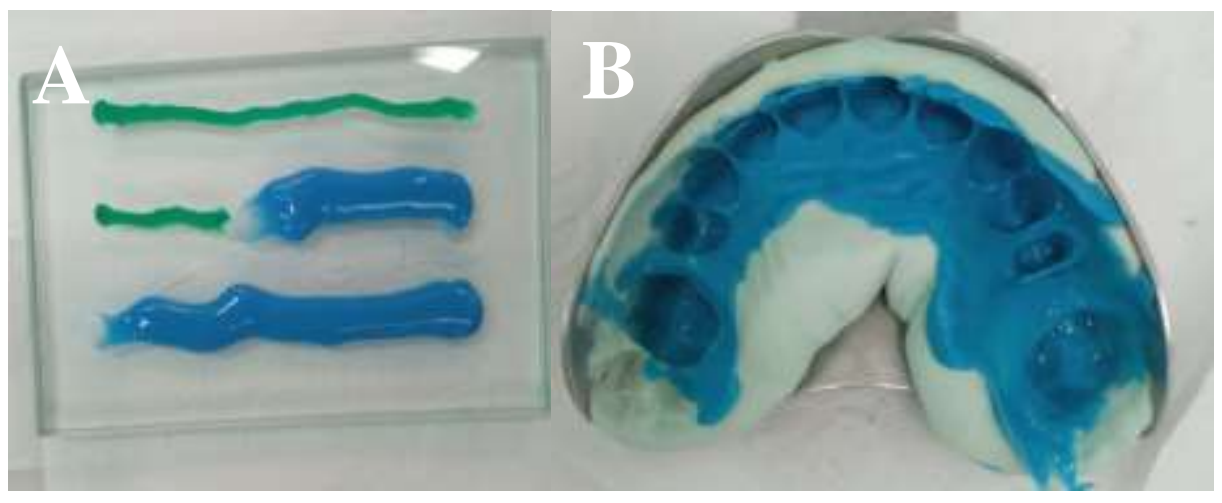

Fonte: Autores.

Assim do término da moldagem, foi realizado o registro de mordida e moldagem da arcada inferior como antagonista, para isso o procedimento teve como material o próprio silicone de condensação pasta pesada, colocado em posição oclusal de todos os elementos da boca da paciente e instruída para ocluir, assim ficou registrado a mordida (Figura 13). Posteriormente foi realizada a moldagem do antagonista com alginato, vazando ambos os modelos com gesso especial tipo IV (Dent Mix, Asfer, Brasil). 
Figura 13: Registro de mordida em silicone (Perfil, Coltene, Brasil)

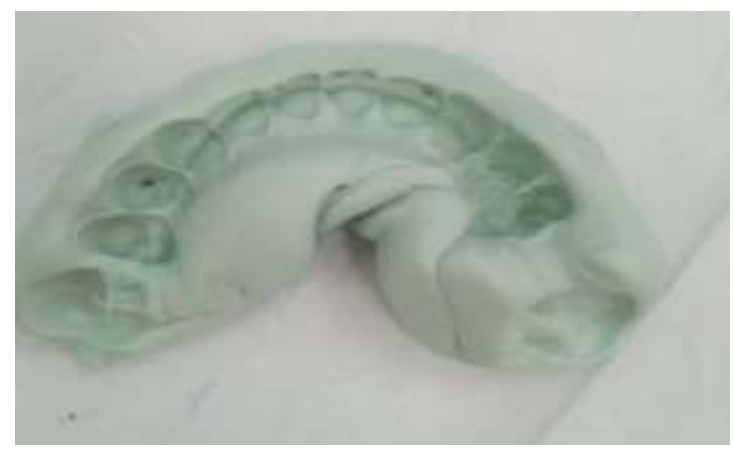

Fonte: Autores.

Com auxílio de uma escala de cor (Classical, VITA, Brasil) foi selecionada em conjunto com a paciente a cor A3 na região cervical e A2 nos terços médio e apical. Foi feita a documentação fotográfica e enviada as informações para o laboratório responsável pela confecção da coroa em dissilicato de lítio (figura 14).

Figura 14: A) Escala de cor (Classical, VITA, Brasil); B) Seleção da cor.

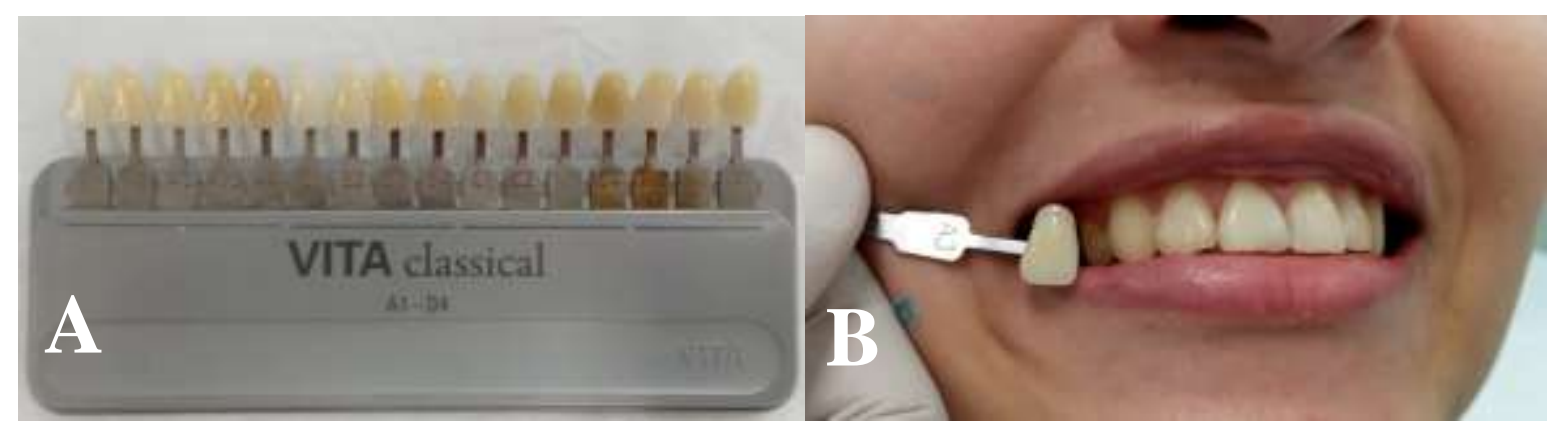

Fonte: Autores.

Após o retorno e confecção da coroa definitiva, foi feito a remoção da coroa provisória e limpeza do preparo com auxílio de água e pedra pomes, logo após realizada a prova da coroa afim de verificar a necessidade de algum tipo de ajuste (figura 15).

Figura 15: Preparo para coroa

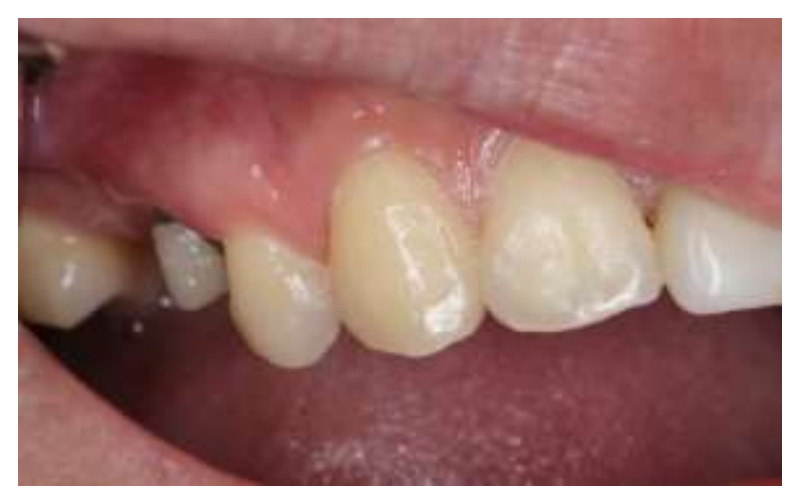

Fonte: Autores.

Após o término de limpeza, foi iniciado o protocolo do condicionamento de superfície da cerâmica a ser cimentada (IPS E.max Press MT, Ivoclar Vivadent). Inicialmente realizado o condicionamento com Ácido fluorídrico a 10\%, (Condac, 
FGM, Brasil), preenchendo toda a parte interna da coroa, como mostra a (Figura 16), após um tempo de espera de aproximadamente 20 segundos foi feito a remoção com água abundante por um tempo de 1 minuto aproximadamente.

Figura 16: Condicionamento com Ácido fluorídrico a 10\%, (Condac, FGM, Brasil).

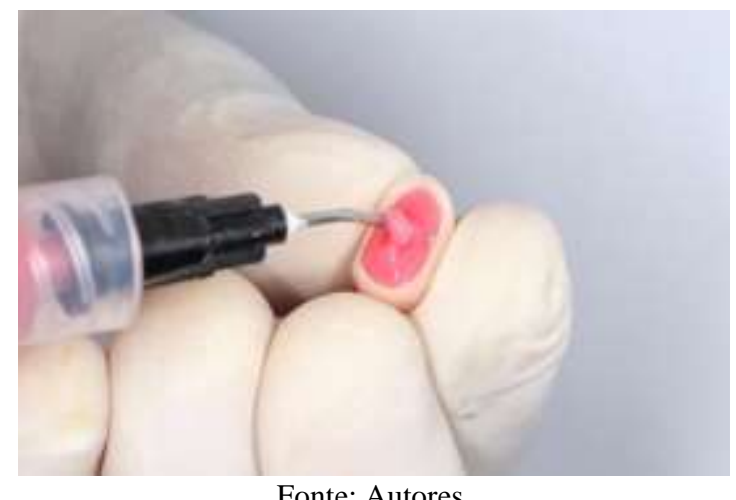

Fonte: Autores.

Logo em seguida foi realizada a limpeza da superfície e secagem, observando no interior da peça um aspecto fosco característico do preparo cerâmico. Posteriormente realizamos a aplicação do agente de união (Silano, Angelus, Brasil) no interior da coroa com uma ponta fina de microbrush (Cavibrush, FGM, Brasil). Ao término do tempo de volatização de aproximadamente 1 minuto, uma leve camada de adesivo universal (Ambar, FGM, Brasil) foi aplicada e aguardado um tempo de volatização por cerca de um minuto (Figura 17).

Figura 17: A) Aplicação do agente de união (Silano, Angelus, Brasil); B) Aplicação do sistema adesivo (Ambar, FGM, Brasil).

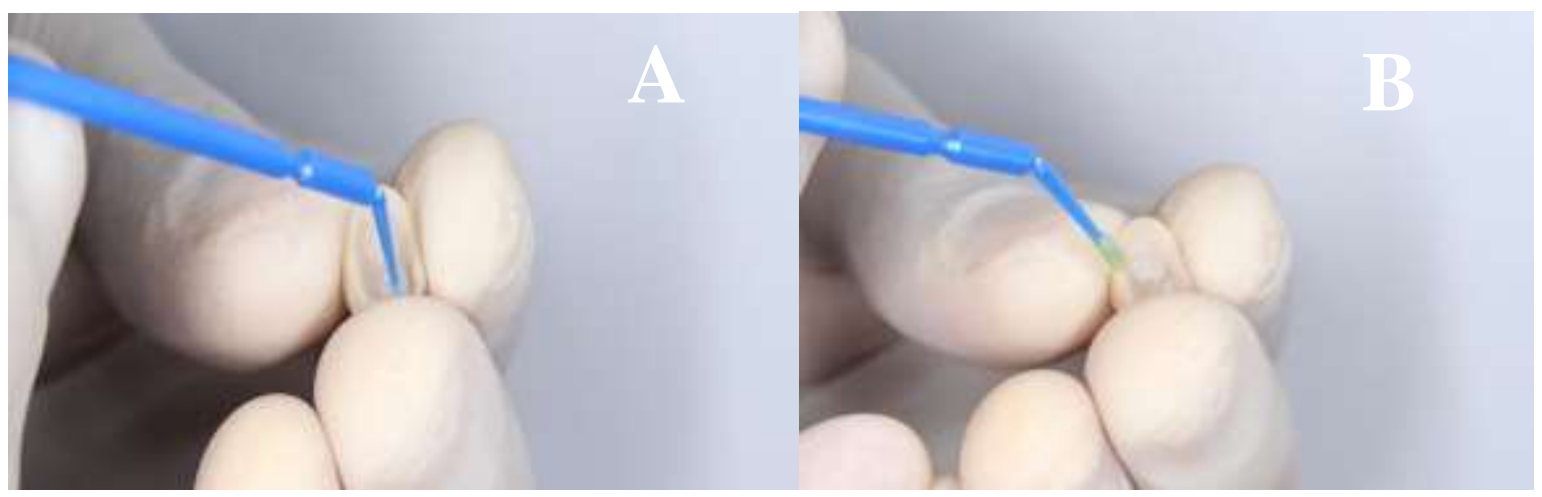

Fonte: Autores.

Posteriormente, demos início ao condicionamento da superfície dental, realizando o condicionamento com ácido fosfórico a 37\% (Condac 37, FGM, Brasil) no preparo dental, aguardado tempo de 30 segundos, realizando a lavagem pelo dobro do tempo e feito a secagem com papel absorvente. Demos início a aplicação do sistema adesivo universal (Ambar, FGM, Brasil) com auxílio do microbrush, friccionando bem toda a superfície e aguardado o tempo de volatização e polimerização (Figura 18). 
Figura 18: A) Condicionamento com ácido fosfórico a 37\% (Condac 37, FGM, Brasil); B) Condicionamento com ácido fosfórico a $37 \%$ (Condac 37, FGM, Brasil).

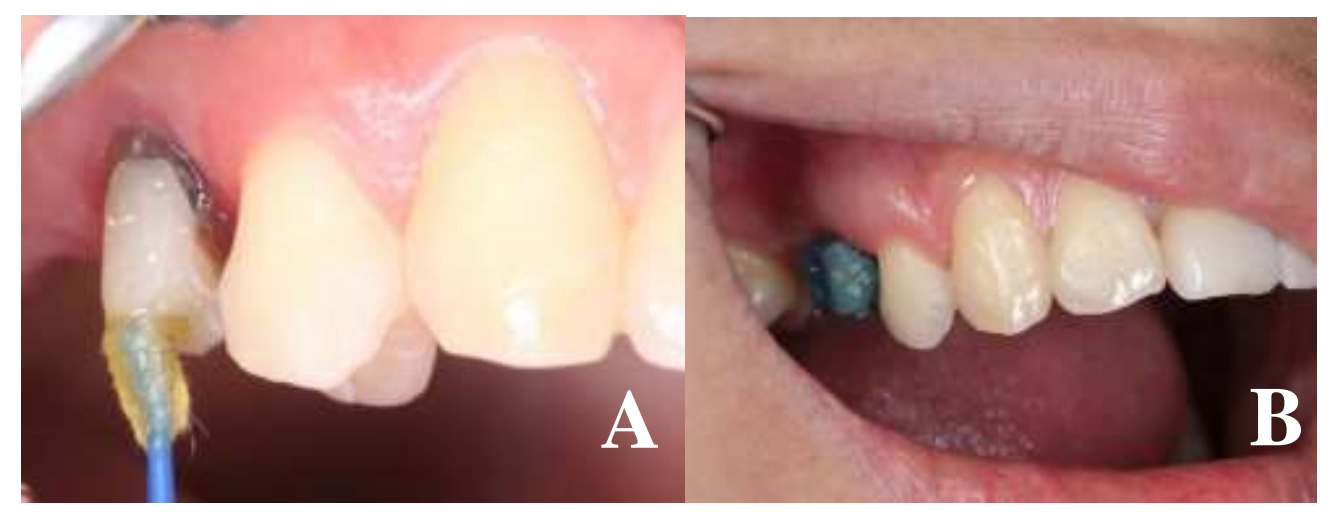

Fonte: Autores.

Já finalizando os protocolos de condicionamento, foi feito a aplicação do cimento resinoso dual de cor A2 (seT PP, SDI, Australia) (Figura 19), e levado ao preparo e acomodado adequadamente, após a adaptação foi necessário a remoção dos excessos com o auxílio de um pincel e aguardado um tempo necessário de 2 a 5 minutos aproximadamente, para polimerização do material.

Figura 19: Aplicação do Cimento A2(seT PP, SDI, Australia).

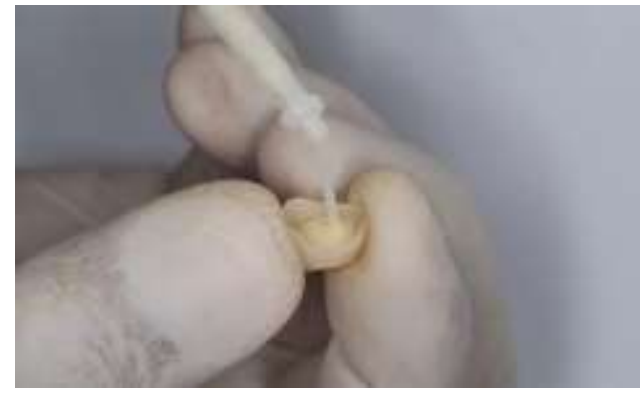

Fonte: Autores.

Finalizado todo o processo de cimentação e aguardado o tempo necessário, não foi necessário nenhum ajuste posterior a cimentação da prótese fixa e foram realizadas as fotografias finais (Figura 20).

Figura 20: A) Face Oclusal; B) Face Vestibular.

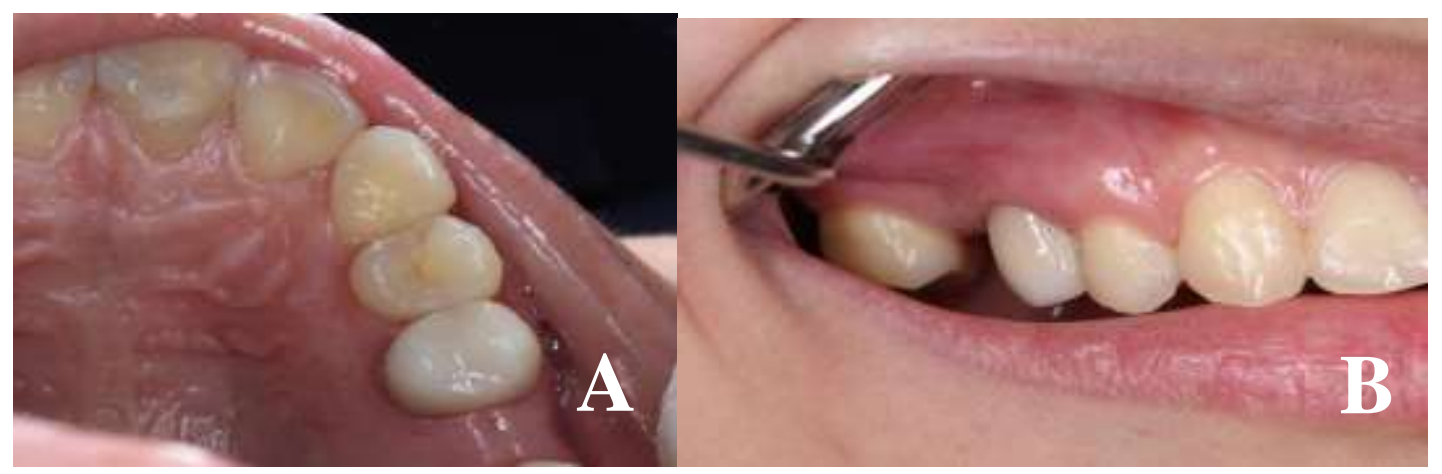

Fonte: Autores. 


\section{Discussão}

A busca pela estética é um dos principais objetivos dos pacientes que procuram os consultórios odontológicos. Partindo-se desse princípio, a reabilitação de dentes do modo indireto deve ser feita de forma criteriosa e responsável, para que o resultado seja duradouro, funcional e com aspecto de naturalidade (Lima, Carvalho \& Cravo, 2010).

Para elementos dentários com grande destruição de estrutura coronária, o uso de retentores intrarradiculares se faz necessário (Faria et al., 2011).

Inúmeros fatores podem influenciar na seleção de um pino intrarradicular como o comprimento, que tem relação com a dissipação da carga de estresse ao longo da raiz; o diâmetro do pino, que deve ser de um terço do diâmetro da raiz; e suas características superficiais (Peroz et al., 2005).

Os agentes de união presentes no silano são capazes de aumentar a molhabilidade e a energia de superfície do PFV, além de promover ligações químicas entre os componentes orgânicos do cimento resinoso e os componentes inorgânicos do pino, como a sílica das fibras de vidro (Goracci et al., 2005).

Os cimentos resinosos devem ser utilizados, preferencialmente, porque promovem maior retenção dos pinos e, consequentemente, aumento da resistência à fratura em comparação aos cimentos de fosfato de zinco (Peroz et al., 2005).

A associação entre pino de fibra de vidro e cerâmica restabelece os princípios de biomimetismo de estrutura dental, por apresentar características como resistência ao desgaste e à compressão, lisura de superfície, brilho e biocompatibilidade com o tecido periodontal (Madureira et al., 2017).

Para a seleção da cerâmica a ser utilizada em cada caso clínico, vários fatores devem ser considerados na escolha, como: magnitude das forças de mastigação incidentes sobre a região a ser reabilitada, oclusão do paciente, cor dos dentes adjacentes e da pastilha cerâmica, características ópticas e de resistência do material, condições clínicas dos tecidos adjacentes e do remanescente dentário (Lima et al., 2013).

O sistema cerâmico IPS E-max apresenta-se como alternativa moderna e inovadora, é composto por cristais de dissilicato de lítio que são embebidos e unidos à matriz vítrea, numa proporção variando de 60 a 70\% em volume de cristais. Apresenta uma estrutura com boa translucidez, que reflete muito bem a luz, devido ao baixo índice de refração dos cristais de Dissilicato de Lítio (Culp \& Mclaren, 2010; Carvalho et al., 2012).

Vale ressaltar que eficácia do tratamento da superfície está relacionada com a composição da cerâmica (Valle et al., 2010).

A seleção do cimento resinoso dual é justificada pela associação de ativação química e de fotoativação, oferecendo maior segurança de polimerização do cimento onde a luz não alcança, e assim espera-se melhora significativa das propriedades físicas e mecânicas (GuedeS et al., 2008). Tal associação contribui para um melhor desempenho da retenção das peças protéticas, quando comparado com os cimentos tradicionais (Namoratto et al., 2013).

A matriz orgânica da resina, o cimento e a sílica presentes na fibra de vidro dos pinos são quimicamente ligados e fornecem melhor capacidade de umedecimento da superfície pelo agente de cimentação (Simões et al., 2016).

\section{Considerações Finais}

O presente estudo concluiu que a reabilitação proposta com o uso de pino de fibra de vidro e a coroa metal free, obtiveram excelentes resultados, pois foram capazes de devolver não só a função, mas a saúde e estética para a paciente, na qual a mesma encontra-se completamente satisfeita com os resultados alcançados, assim também pôde ser observado nas imagens os resultados. 


\section{Referências}

Alomari, Q. D., Barrieshi, K. M., \& Al-Awadhi, A. (2011). Effect of post length and diameter on remaining dentine thickness in maxillary central and lateral incisors. Int Endod J, 44(10), 956-66.

Amarnath G. S., Pandey, A., Prasad, H. A., \& Hilal, M. (2015). Comparative Evaluation of Enhancing Retention of Dislodged Crowns Using Preparation Modifications and Luting Cements: An In-Vitro Study. J Int Oral Health. 7(8), 47-51.

Baratieri, L. N., et al. (2001). Odontologia Restauradora - Fundamentos e Possibilidades. Livraria Santos Editora, 16, 619-672.

Bitter, K., Noetzel, J., Stamm, O., Vaudt, J., Meyer-Lueckel, H., Neumann, K., et al. (2009). Randomized clinical trial comparing the effects of post placement on failure rate of postendodontic restorations: preliminary results of a mean period of 32 months. J Endod. 35(11), 1477-82.

Büttel, L., Krastl, G., Lorch, H., Naumann, M., Zitzmann, N. U., \& Weiger, R. (2009). Influence of post fit and post length on fracture resistance. Int Endod J. 42(1), 47-53.

Cheung, W. (2005). A review of the management of endodontically treated teeth. Post, core and the final restoration. J Am Dent Assoc. 136(5), 611-9.

Costa, D. M. C., Prado, M., Costa, V. S., Gaiotte, M., Simão, R. A., \& Bastian, F. L. (2012). Comparison between the effect of plasma and chemical treatments on fiber post surface. J Endod, ;38(2), 215-8. http://dx.doi.org/10. 1016/j.joen.2011.10.020. PMid:22244639.

Culp, L., \& Mclaren, E. A. (2010). Lithium disilicate: the restorative material of multiple options. Compendium, 31(9), 716-725.

Eskitasciolglu, G., Belli, S., \& Kalkan, M. (2002). Evaluation of two post core systems using two different methods (fracture strength test and a finite elemental stress analysis). J Endod, 28(9):629-33.

Faria A. C. L., Rodrigues R. C. S., de Almeida Antunes, R. P., de Mattos, M. D. G. C., \& Ribeiro, R. F. (2011). Endodontically treated teeth: characteristics and considerations to restore them. J prosthodontic Research, 55(2), 69-74.

Farina A. P., Cecchin, D., Garcia, L. D. A. F., Naves, L. Z., Sobrinho, L. C., \& Pires-De-Souza, F. D. E. C. (2011). BondStrength of fiber posts in different root thirds using resin cement. J Adhes Dent. 13(2), 179-86.

Fernandes A. S., Shetty, S., \& Coutinho, I. (2003). Factors determining post selection: A literature review. J Prosthet Dent, 90(6), 556-62.

Fernandes Junior, D., \& Beck, H. (2016). Vantagens dos pinos de fibra de vidro. Revista de Odontologia da UBC, 6(1).

Freitas, A., Rossi, A., Brene, C. G., \& Rebello, I. C. (2014). Planejamento em Reabilitação Oral - Estética do Sorriso em reabilitação protética. Editora Napoleão $-1^{\text {a }}$ edição cap. 1,300 .

Furtos, G., Baldea, B., \& Silaghi-Dumitrescu, L. (2016). Development of new radiopaque glass fiber posts. Mater Sci Eng C Mater Biol Appl. 59, 855-62.

Goracci, C., Raffaelli, O., Monticelli, F., Balleri, B., Bertelli, E., \& Ferrari, M. (2005). The adhesion between prefabricated FRC posts and composite cores: microtensile bond strength with and without post silanization. Dent Mater, 21(5), 437-44.

Guedes, L. L. S., Matto, E. C. G., Zani, S. M., Prates, L. H. M., \& Chain, M. C. (2008). Avaliação das propriedades mecânicas de cimentos resinosos convencionais e autocondicionantes. Rev Odontol UNESP. 37(1), 85-9.

Jiangkongkho, P., Kamonkhantikul, K., Takahashi, H., \& Arksornnukit, M. (2013). Fracture resistance of endodontically treated teeth using fiber post with an elastic modulus similar to dentin. Dent Mat J, 32(5), 781-6.

Kaya, B., \& Ergun, G. (2013). The effect of post length and core material on root fracture with respect to different post materials. Acta Odontol Scand. 71(5): 1063-1070. Doi: 10.3109/00016357.2012.741706.

Lima, A. F., Carvalho, J. F. O., Cravo, F. L. (2010). Restaurações cerâmicas em dentes anteriores: simples realização? Rev Dental Press Estét,7(4), 88-96.

Lima, R. B. W., Figueiredo, R. J. A., De Andrade, A. K. M., Duarte, R. M. (2013). Otimizando a Estética do Sorriso através de Coroa Cerâmica "Metal Free” relato de caso. Rev Bras de Ciências da Saúde. 17(2), 165-70.

Madureira, I. T., Bem, J. S. P., De Andrade, Veras, S. R., Lins, C. C. D. S. A., Da Costa Leite, E. B. (2017). Reabilitação estética anterior com núcleos de pinos de fibra de vidro e coroas metal free: relato de caso clínico. Odontol Clín-Cient, 16(1)57-60.

Moro, A. A. M., \& Matsumoto, W. (2005). Núcleos metálicos fundidos x pinos pré-fabricados. Revista Ibero-americana de Prótese Clínica e Laboratorial. 7(36), 167-72.

Muniz, L., Góes, C. F., Oliveira, A. C. P. C., Mathias, P., Bezerra, R. B., |\& Fontes, C. M. (2005). Restaurações diretas associadas a pinos de fibra de vidro em dentes fraturados. Relato de caso clínico. Rev Dental Press, 2(3), 45-47.

Namoratto, L. R., Ferreira, R. D. S., Lacerda, R. A. V., Sampaio Filho, H. R., \& Ritto, F. P. (2013). Cimentação em cerâmicas: evolução dos procedimentos convencionais e adesivos. Rev Bras Odontologia, 70(2), 142-7.

Nova, V., Karygianni, L., Altenburger, M. J., Wolkewitz, M., Kielbassa, A. M., \& Wrbas, K. T. (2013). Pull-outbond strength of a fibre-reinforced composite post system luted with self-adhesive resin cements. J Dent, 41(11), 1020-6.

Oliveira. A. A. (2013). Entender, planejar, executar: o universo das restaurações estéticas. Nova Odessa: Napoleão 2. Ed. São Paulo: Artes Médicas. Cap. 5. 2. Cap. 6,178-214.

Pedreira, A. P. R. V., \& Koren, A. R. R. (2013). Quando indicar retentores intrarradiculares de fibra de vidro ou metálicos? Oral Sciences; 5(2), 3-4. 
Research, Society and Development, v. 10, n. 16, e19101623601, 2021

(CC BY 4.0) | ISSN 2525-3409 | DOI: http://dx.doi.org/10.33448/rsd-v10i16.23601

Pegoraro, L. F. (2012). Prótese fixa: bases para o planejamento em reabilitação oral. Prótese fixa [recurso eletrônico]: bases para o planejamento em reabilitação oral / Luiz Fernando Pegoraro.

Peroz, I., Blankenstein, F., Lange, K. P., \& Naumann, M. (2005). Restoring endodontically treated teeth with posts and cores-a review. Quintessence Int, 36(9):737-46.

Pilo, R., Shapenco, E., \& Lewinstein, I. Residual dentin thickness in bifurcated maxillary first premolars after root canal and post space preparation with parallel-sided drills. J Prosthet Dent. 2008 Apr;99(4):267-73.

Sedgley, C. M., \& Messer, H. H. (1992). Are endodontically treated teeth more brittle? J Endod. 18(7), 332-5.

Simões, T. C., Luque-Martinez, Í., Moraes, R. R., Sá, A., Loguercio, A. D., \& Moura, S. K. (2016). Longevity of bonding of selfadhesive resin cement to dentin. Oper Dent, 41(3), e64-72. http://dx.doi.org/10.2341/14-266-LR. PMid:26918926.

Sirimai, S, Riis, D. N., \& Morgano, S. M. (1999). An in vitro study of the fracture resistance and the incidence of vertical root fracture of pulpless teeth restored with six post-and-core systems. J Prostht Dent, 81(3), 262-9.

Skupien, J. A., Sarkis-Onofre, R., Cenci, M. S., Moraes, R. R., \& Pereira-Cenci, T. (2015). A systematic review of factors associated with the retention of glass fiber posts. Braz Oral Res. 29(1), 1806-83242015000100401.

Trushkowsky, R. D. (2011). Esthetic and functional consideration in restoring endodontically trated teeth. Dent Clin North Am. 55(2), 403-10.

Valle, A. L., Martin, L. M., Chidiak-Tawil, R., Pimentel, G. H. D., Rodrigues, M. G. S., \& Ramos, M. B. (2010). Sistemas cerâmicos atuais: revisão de literatura. Rev Dental Press Estética.7(1), 106-7.

Zhou, L., \& Wang, Q. (2013). Comparison of fracture resistance between cast posts and fiber posts: a meta-analysis of literature. J Endodontics. 39(1), 11-5. 